\title{
Simulation studies of self-assembly of end-tethered nanorods in solution and role of rod aspect ratio and tether length
}

\author{
Mark A. Horsch and Zhenli Zhang \\ Department of Chemical Engineering, University of Michigan, Ann Arbor, Michigan 48109-2136 \\ Sharon C. Glotzer ${ }^{\mathrm{a})}$ \\ Department of Chemical Engineering, University of Michigan, Ann Arbor, Michigan 48109-2136 \\ and Department of Materials Science and Engineering, University of Michigan, Ann Arbor, \\ Michigan 48109-2136
}

(Received 1 September 2006; accepted 21 September 2006; published online 9 November 2006)

\begin{abstract}
We present temperature versus concentration phase diagrams for "shape amphiphiles" comprised of tethered moderate and low aspect ratio rods. Simulations of moderate aspect ratio rods (first reported by Horsch et al. [Phys. Rev. Lett. 95, 056105 (2005)]) predict their self-assembly into spherical micelles with bcc order, long micelles with nematic order, a racemic mixture of hexagonally ordered chiral cylinders, two perforated phases: one with tetragonal order and one with hexagonal order, and a smectic C lamellar phase. In contrast, we predict here that small aspect ratio tethered rods self-assemble into bcc ordered spherical micelles, hexagonally ordered cylinders, and a smectic $\mathrm{C}$ lamellar phase. We compare and contrast the phases obtained for the two aspect ratios and examine in further detail several unusual phases. Our simulations also reveal that for moderate aspect ratio rods there is a tendency toward phases with decreasing interfacial curvature with decreasing coil size, including a double gyroid phase. In addition, we investigate the role of tether length on the assembled structures. Our results are applicable to short rod-coil block copolymers and rodlike nanoparticles with polymer tethers, and to colloidal building blocks comprised of a flexible string of colloids tethered to a rigid string of colloids, with the interactions scaled appropriately. (c) 2006 American Institute of Physics. [DOI: 10.1063/1.2363983]
\end{abstract}

\section{INTRODUCTION}

Self-assembly is a promising strategy by which nanometer sized building blocks (NBBs) can be manipulated to form useful structures for a variety of applications. ${ }^{1}$ Functionalization of the NBBs with polymer tethers provides a means through which the NBBs can be induced to selfassemble into target structures. ${ }^{2-5}$ Polymer-tethered nanoparticles comprised of a flexible solvent-loving part and a rigid solvent-hating part constitute a class of "shape amphiphiles." 4,6 These new materials have much in common with traditional molecular amphiphiles but here the "head group" may be a hard nanometer sized particle. Under the right conditions, immiscibility between the tether and particle and the geometric packing of the hard particles combine to produce complex self-assembled structures. Conventional rod-coil block copolymers can be considered as one limit of polymer-tethered rods (PTRs), a specific class of shape amphiphiles in which the nanoparticle is rodlike. Various architectures of rod-coil block copolymers have been synthesized, with flexible polymers bonded to the middle of the rod, to each end of the rod, or at only one end of the rod. ${ }^{7}$ Rich morphologies arising from their self-assembly have been observed experimentally, e.g., layered smectic, ${ }^{8}$ wavy, ${ }^{8}$ zigzag, ${ }^{9}$ honeycomb, ${ }^{10,11}$ and tetragonally perforated lamellae, ${ }^{10,11}$

\footnotetext{
${ }^{a)}$ Author to whom correspondence should be addressed. Electronic mail: sglotzer@umich.edu
}

hexagonally packed cylinders, ${ }^{11}$ bicontinuous cubic structures, ${ }^{12}$ and hollow spherical and cylindrical micelles. ${ }^{13}$

In contrast to block copolymers (BCPs) consisting of flexible blocks, theoretical progress is less developed for rodcoil BCPs. Although the same principles govern the morphologies that form, the entropic interactions are more complicated in the latter due to the asymmetry between the two blocks and the tendency of the rigid block to orient itself relative to other rigid blocks as in molecular liquid crystals. Halperin ${ }^{14}$ and Semenov and Vasilenko ${ }^{15}$ used scaling analysis to study the smectic A and smectic C phases. Williams and Fredrickson postulated the formation of pucklike micelles when the volume fraction of the coil is high. ${ }^{16}$ They proposed that within the pucks bundles of rods form a truncated cylindrical structure. Holyst and Schick used a Landau free energy expansion considering both compositional and orientational order parameters to study the isotropic nematic transition and the smectic phases near the order disorder transition (ODT). ${ }^{17}$ Matsen and Barett performed selfconsistent field theory (SCFT) calculations on rod-coil block BCPs. ${ }^{18}$ They used Flory's lattice theory to incorporate the orientational interactions and their study was limited to the nematic and smectic phases. Prymitsyn and Ganesan presented a SCFT model where the orientational interactions were treated using the Maier-Saupe potential. ${ }^{19}$ Using this model, they were able to predict the phase diagram for rodcoil BCPs for one and two dimensions and their predictions were qualitatively similar to several experimental findings. 
Recently, Chen et al. introduced a new SCFT model to study the effects of the rod fraction as it pertains to the selfassembly of the model rod-coil copolymers. ${ }^{20}$ Their work was the first time that SCFT predicted the hexagonal cylinder phase for rod-coil BCPs. Our previous work ${ }^{4}$ focused on the self-assembly of tethered nanorods in a selective solvent and we predicted for the first time the formation of the tetragonally and hexagonally perforated lamellar phases as well as identifying a novel hexagonally packed racemic phase of chiral cylinders. The tetragonal phases have been observed in experimental systems where the rod is solvophobic and the tether solvophilic ${ }^{21}$ but had not yet been realized by either theory or simulation. Here, we extend our previous work and demonstrate the effect of both the degree of polymerization of the tethers and the aspect ratio of the rods on the selfassembled structures. We also elaborate on each of the morphologies presented in our previous study. ${ }^{4}$

Using a coarse-grained, particle-based model $^{2}$ and Brownian dynamics (BD) we study the phase behavior of a solution of tethered rods in three dimensions. We compare the phase behavior of high and low aspect ratio rods in the temperature versus concentration plane. For moderate aspect ratio tethered rods, we also study the effect of the relative coil fraction, $f_{\text {coil }}$. A primary advantage of this particle-based model is that the liquid crystal behavior of the rods and the complex entropic interactions arising from the asymmetry between the rigid rods and the flexible tethers are automatically incorporated. Another important feature is that these simulations are performed in three-dimensional (3D) space. Our previous ${ }^{4}$ study demonstrated that due to the coupling between orientational ordering and chain stretching, the number of dimensions is significant in determining the final structures, especially the local packing of the rods. The manuscript proceeds as follows. The model and simulation method are described in Sec. II. The temperature versus concentration phase diagram for moderate aspect ratio rods is described in Sec. III. The effects of relative coil fraction for moderate-aspect ratio rods are discussed in Sec. IV. The temperature versus concentration phase diagram for low aspect ratio rods is discussed in Sec. V, and a discussion of all results can be found in Sec. VI.

\section{MODEL AND METHOD}

\section{A. Model}

\section{Rods}

We model the rods as a series of $N_{r b}$ beads linked rigidly in a linear geometry, ${ }^{2}$ see Fig. 1 . The degree of roughness within a rod is determined by the spacing $d$ between adjacent spheres. As $d \Rightarrow 0$ and $N_{r b} \Rightarrow \infty$ the rods become perfectly smooth spherocylinders. The length of the rod, $L$, is defined as $\left[\left(N_{r b}-1\right)(d)+1\right]$. The rods used in our study are not intended to represent a specific chemistry but are intended to capture the universal behavior of rodlike particles permanently connected to a flexible tether, where the size of the tether is on the order of the size of the rod. Our model also applies to colloidal rods formed by fusing a series of colloidal beads into a linear geometry. The interaction potentials described below can be easily modified for such systems.

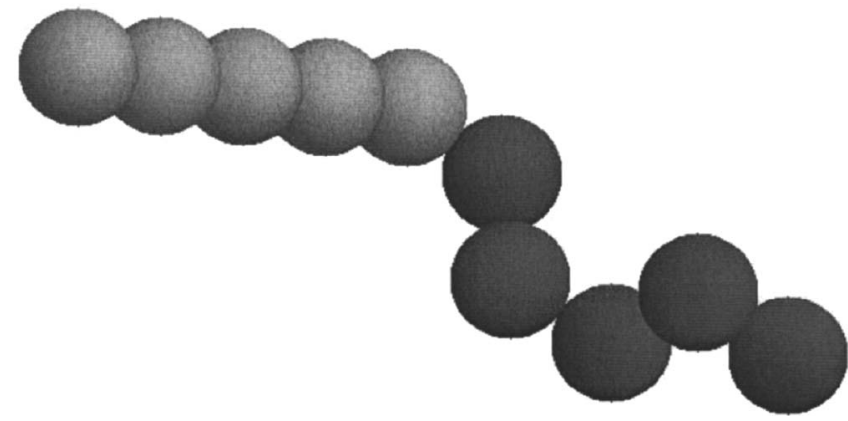

FIG. 1. Rendering of model tethered nanorod.

\section{Tethers}

The flexible tethers are modeled as linear, bead-spring chains comprised of $N_{t b}$ beads bonded together via a finitely extensible, nonlinear, elastic (FENE) spring. ${ }^{22}$

$$
U_{\mathrm{FENE}}= \begin{cases}-0.5 k R_{\mathrm{o}}^{2} \ln \left|1-\left(\frac{r}{R_{\mathrm{o}}}\right)^{2}\right|, & r<R_{\mathrm{o}} \\ \infty, & r \geqslant R_{\mathrm{o}},\end{cases}
$$

where $r$ is the distance between neighboring beads and $k$ and $R_{0}$ are the energy and length parameters of the potential, respectively. As in prior works, their values are taken as $k$ $=30$ and $R_{0}=1.5 \sigma$, respectively. These values ensure relatively stiff bonds and prevent unphysical chain crossings.

\section{Modeling amphiphilic behavior}

To study self-assembly resulting from solvent selectivity, we use the following definition for poor and good solvents. If the solvent is poor for a particular species, the nonbonded interactions between that species are modeled with a potential containing an attractive component to incorporate immiscibility. If the solvent is good for a particular species the nonbonded interactions between that species are modeled with a purely repulsive interaction potential. In this study, we focus on tethered rods in a solvent that is poor for the rods. The miscibility of the nanorods is implemented via a truncated and shifted, site-site, 12-6 Lennard-Jones (LJ) potential:

$$
\begin{aligned}
U_{\mathrm{LJ}}(r)= & \frac{4 \varepsilon}{k_{B} T}\left[\left(\frac{\sigma}{r}\right)^{12}-\left(\frac{\sigma}{r}\right)^{6}\right]-\frac{4 \varepsilon}{k_{B} T}\left[\left(\frac{\sigma}{r_{c}}\right)^{12}-\left(\frac{\sigma}{r_{c}}\right)^{6}\right] \\
& -\left(r-r_{c}\right)\left(\frac{d U_{\mathrm{LJ}}(r)}{d r}\right)_{r=r_{c}}, \quad r \leqslant r_{c},
\end{aligned}
$$

where $r_{c}$ is the cutoff radius and is taken to be $2.5 \sigma$. Nonbonded interactions between tethers and between tethers and rods are treated with a Weeks-Chandler-Andersen (WCA) potential. The WCA potential is the 12-6 LJ potential truncated at the minimum and shifted vertically by $\varepsilon / k_{B} T$, giving a purely repulsive potential:

$$
U_{\mathrm{WCA}}(r)=\frac{4 \varepsilon}{k_{B} T}\left[\left(\frac{\sigma}{r}\right)^{12}-\left(\frac{\sigma}{r}\right)^{6}\right]-\frac{\varepsilon}{k_{B} T}, \quad r \leqslant r_{c}^{\prime},
$$

where $r_{c}^{\prime}=2^{1 / 6} \sigma$. The length and energy scales for these systems are $\sigma$ and $\varepsilon$, respectively, with the time unit $\tau$ $=\sigma \sqrt{m / \varepsilon}$, where $m$ is the mass of a bead, $\sigma=\sigma_{\mathrm{TT}}=\sigma_{\mathrm{RR}}$ 
$=\sigma_{\mathrm{RT}}$ is the diameter of a bead, and $\varepsilon=\varepsilon_{\mathrm{TT}}=\varepsilon_{\mathrm{RR}}=\varepsilon_{\mathrm{RT}}$ are the interaction parameters. The subscripts TT, RR, and RT correspond to tether-tether, rod-rod, and rod-tether, respectively. The dimensionless temperature is in units of $\varepsilon / k_{B} T$.

\section{B. Simulation method}

$\mathrm{BD}$, a mesoscopic simulation technique, is used to study the model, tethered rods. Although solvent particles are not explicitly included in this method, certain solvent effects are treated implicitly allowing for the study of the model NBBs in solution. The trajectory of each "bead" is governed by the Langevin equation:

$$
m_{i} \ddot{\mathbf{r}}_{i}=-\gamma_{i} \mathbf{y}_{i}+\mathbf{F}_{i}^{C}(\mathbf{r}(t))+\mathbf{F}_{i}^{R}(t),
$$

where $m_{i}$ is the mass of bead $i$, and $\mathbf{r}_{i}, \mathbf{v}_{i}, \mathbf{F}_{i}^{C}$, and $\gamma_{i}$ represent the position, velocity, conservative force vectors, and the friction coefficient of bead $i$, respectively. It is assumed that there are no spatial or temporal fluctuations in the friction coefficient and $\gamma_{i}$ is chosen to be 1.0 , thereby limiting the ballistic motion of a bead to $\sim 1.0 \sigma$. The random force vector, $\mathbf{F}_{i}^{R}$, is assumed to be stationary, Markovian, and Gaussian with zero mean. It is further assumed that there are no temporal correlations in the random force and that it is independent of the conservative force. The amplitude of the random force must satisfy the fluctuation dissipation theorem and therefore must meet the following criteria:

$$
\begin{aligned}
& \left\langle\boldsymbol{F}_{i}^{R}(t)\right\rangle=0, \\
& \left\langle\boldsymbol{F}_{i}^{R}(t) \boldsymbol{F}_{j}^{R}\left(t^{\prime}\right)\right\rangle=6 \gamma k_{B} T \delta_{i j} \delta\left(t-t^{\prime}\right) .
\end{aligned}
$$

The friction coefficient and the random force couple the system to a heat bath, where the latter term acts as a heat source compensating for frictional losses incurred by the particles due to viscous drag. The combination of the random and viscous forces helps to minimize numerical roundoff errors that occur over long simulation runs. The stationary solution to the Langevin equation is the Boltzmann distribution and therefore these simulations sample the canonical $(N V T)$ ensemble.

The rotational degrees of freedom of the rod are incorporated using the equations for rotation of linear bodies. ${ }^{23}$ To integrate the equations of motion we employ the leapfrog integration algorithm. The time step $\Delta t$ used to integrate the discretized equations of motion is $0.01 \tau$. All simulations are initially carried out in a cubic cell with periodic boundary conditions. Systems are equilibrated athermally (repulsive interactions only) and subsequently cooled to the target temperature by decrementing the temperature such that $\Delta T$ $=0.01$. To ensure that the self-assembled structures are independent of the cooling history several different cooling rates are tested. To avoid system size effects, we consider systems of $N_{R}=600,800,1000,1300,2000,4800$, and 6400, which corresponds to $N=6000-140800$ beads depending on the roughness of the rod. $N_{R}$ is the number of rods and $N$ the total number of coarse-grained beads in the system, respectively. We also employ the box search algorithm to allow the shape of the box to change during the course of the simulation. $^{24}$

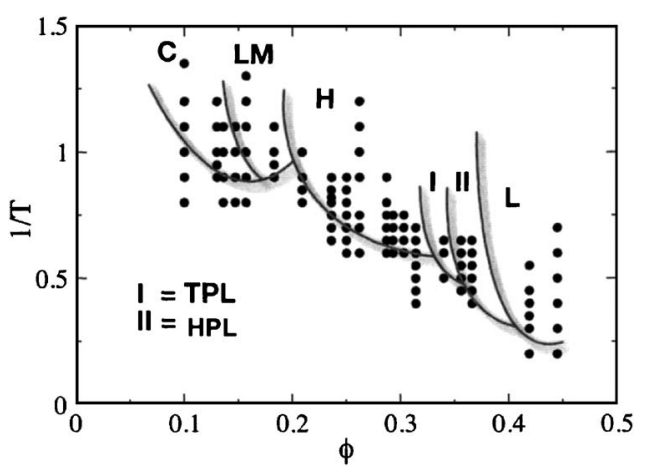

FIG. 2. $T$ vs $\phi$ morphological phase diagram of moderate aspect ratio tethered rods. The solid lines do not represent calculated phase boundaries but are used as visual guides to identify the regions in which specific ordered structures are observed. C, LM, H, TPL, HPL, and L correspond to bcc, nematically ordered long micelles, hexagonally ordered chiral cylinders, tetragonally and hexagonally perforated lamellae, and smectic $\mathrm{C}$ lamellae, respectively.

\section{SELF-ASSEMBLY OF MODERATE ASPECT RATIO TETHERED NANORODS}

In our studies, we define moderate aspect ratio rods as rods with an aspect ratio of greater than 5:1 and low aspect ratio rods as those with an aspect ratio of less than $5: 1$. This definition is chosen based on the ability of systems of hard rods to form a nematic phase when their aspect ratio is greater than $5: 1 .^{25}$ The phase diagram (inverse temperature $1 / T$ versus volume fraction $\phi)$ for a solution of moderate aspect ratio tethered rods is presented in Fig. 2. $\phi$ is defined as $\left[\left(N_{R} \pi \sigma^{2} L / 4\right)+\left(N_{t b}+1\right) \pi \sigma^{3} / 6\right] / V_{\text {box }}$ for smooth rods (rods containing overlapping spheres), and $N \pi \sigma^{3} / 6 V_{\text {box }}$ for rods without overlapping spheres. Below we discuss the details of the predicted phases.

\section{A. Cubic micelle phase $(C)$}

We observe the formation of a cubic micelle phase for volume fractions between $\phi=0.1$ and 0.17 [Fig. 3(a)]. As the system cools micelles form which at sufficient concentrations pack into a bcc morphology. To quantify the shape of the micelles, we calculate the asphericity parameter $A_{s}$ defined as

$$
A_{s}=\frac{\sum_{i>j}^{N_{\mathrm{dim}}}\left\langle\left(R_{i}^{2}-R_{j}^{2}\right)^{2}\right\rangle}{\left(N_{\mathrm{dim}}-1\right)\left\langle\left(\sum_{i=1}^{d} R_{i}^{2}\right)^{2}\right\rangle}
$$

where $N_{\text {dim }}$ is the dimension of the aggregate, here $N_{\text {dim }}=3$. We find that on average the micelles contain 40 tethered rods with $A_{s}=0.05$ indicating that the micelles are spherical in shape. Snapshots of the system reveal that within the micelles the rods pack into a flattened cylinder where the diameter is larger than the height [Fig. 3(b)]. The orientation of the tethers, however, compensates for the disparity between the height and diameter of the rod core and overall the micelles attain a spherical shape [Fig. 3(c)]. System snapshots further reveal that the rods do not pack parallel to each other but that they are tilted [Fig. 3(b)] with respect to the face 

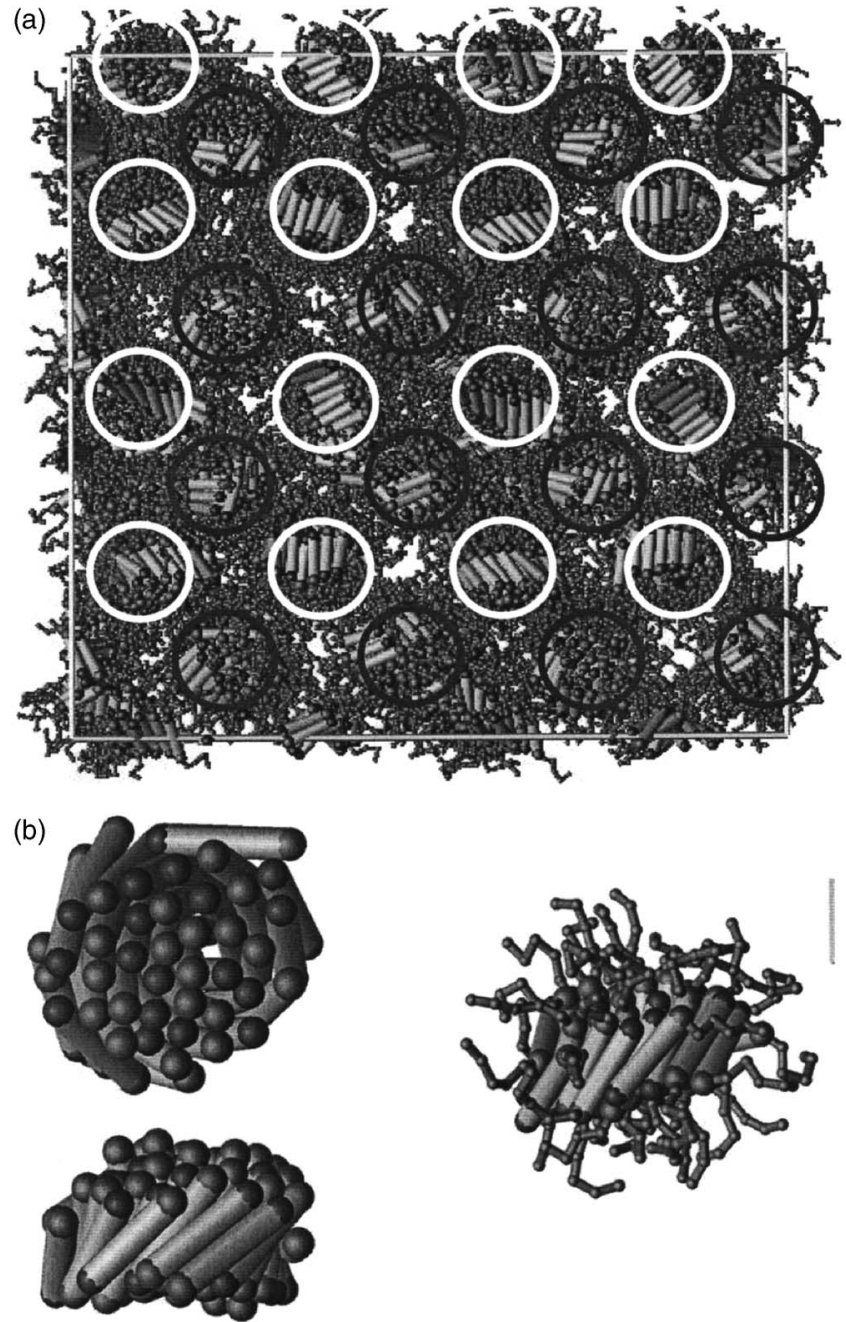

FIG. 3. Simulation snapshots obtained from a system of $N_{r}=4800$ tethered rods at $\phi=0.13$, and $1 / T=1.25$. (a) Example bcc morphology. The circles are guides to the eyes. The blue circles represent micelles in the center of the unit cell and the white circles represent micelles on the corners. The periodic boundary conditions have been removed for visualization. (b) A single micelle, containing 40 tethered rods, extracted from the simulation cell. The tethers have been removed for viewing ease. The left image is the face view of the cylindrical core and the lower image is the side view. The rod ends where tethers are attached are colored red and those without tethers are blue. The right image is the side view of the micelle with the tethers attached. The tethers do not extend radially from the core of the micelle leaving the core partially exposed.

normal of the cylinder. Although we observe that the micelles are spherical in shape, we note that they are not isotropic in regard to intermicellar interactions. The system snapshot in Fig. 3(c) clearly reveals that the tethers do not extend radially from the center of the micelle but that they can be described more accurately as extending normally from the face of the cylinder. Therefore, the intermicellar interactions are dependent on the relative orientation of the micelles-a point that will be discussed further in the following section.

\section{B. Long micelles (LMs)}

Between the cubic micelle and the cylinder phase, 0.17 $\leqslant \phi \leqslant 0.2$, we predict the formation of a nematically ordered long micelle phase [Fig. 4(a)]. Snapshots of the system re-

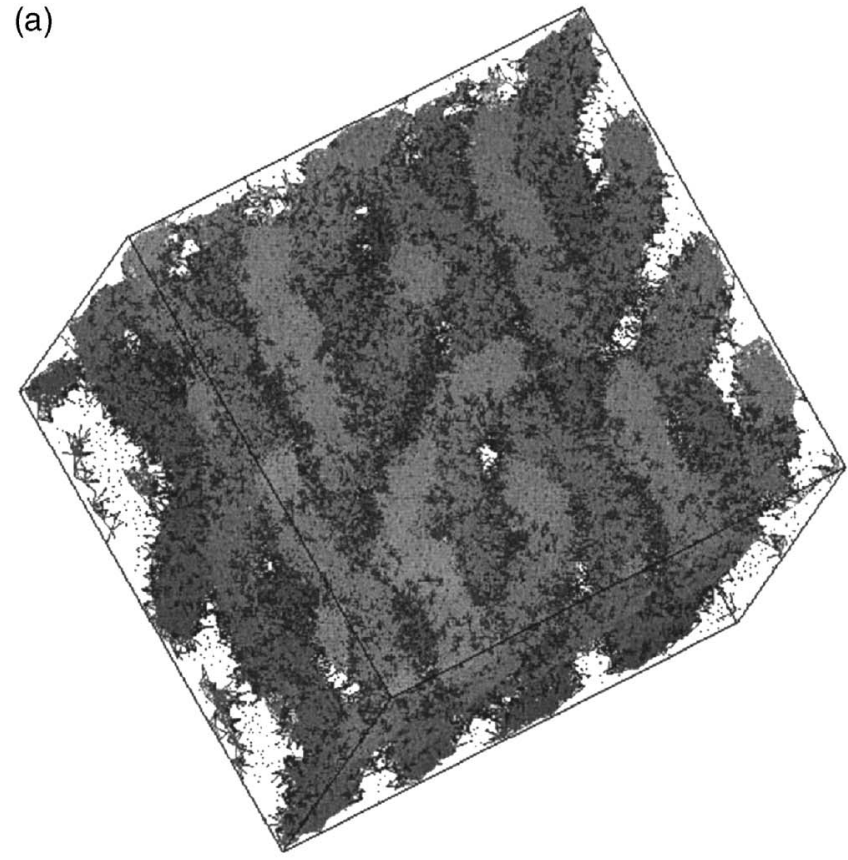

(b)
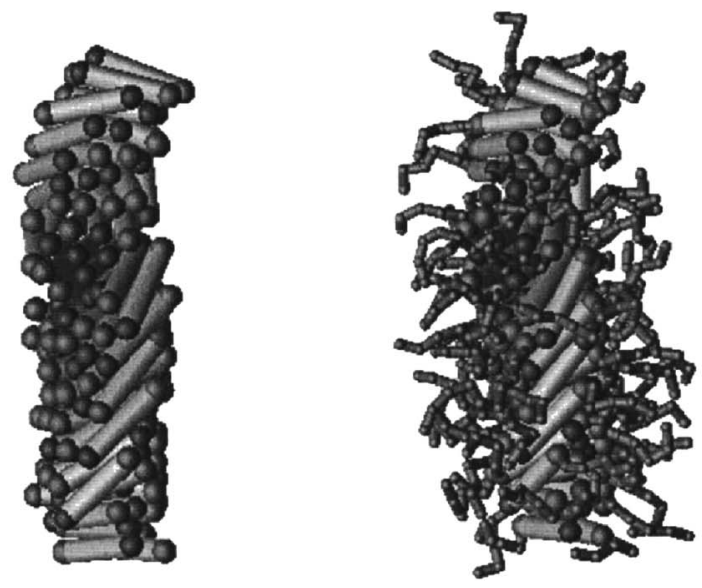

FIG. 4. Simulation snapshots obtained from a system of $N_{r}=4800$ tethered rods at $\phi=0.18$, and $1 / T=0.9$. (a) System snapshot of the nematically ordered long micelle morphology. For visualization purposes the polymer tethers have been removed and the micelles have been rendered as surfaces of constant density. The red specks are used to add contrast to the micelles. (b) A single micelle, containing 111 tethered rods, extracted from the simulation cell in "A." The tethers have been removed in the left image and the rod ends that are attached to tethers are colored red. Rod ends that are not attached to tethers are colored blue. The right image illustrates the packing of the tethers.

veal that within the long micelles the rods pack with a regular twist along the long axis of the micelle [Fig. 4(b)]. To quantify the shape of the micelles, we again use the asphericity parameter. An example of the asphericity parameter as a function of micelle size for $\phi=0.18$, and $1 / T=0.85$ for a system of long micelles is presented in Fig. 5. We postulate that the formation of the long micelle phase arises from the anisotropic intermicellar interactions. As the concentration of the system is increased the core of the micelle cannot stretch to accommodate more amphiphiles per micelle as in the case of flexible amphiphiles and because of the strong tendency for the rods to align the rods cannot pack randomly. However, because of the anisotropic interactions the micelles can reorient themselves such that the rod-rod interactions are 


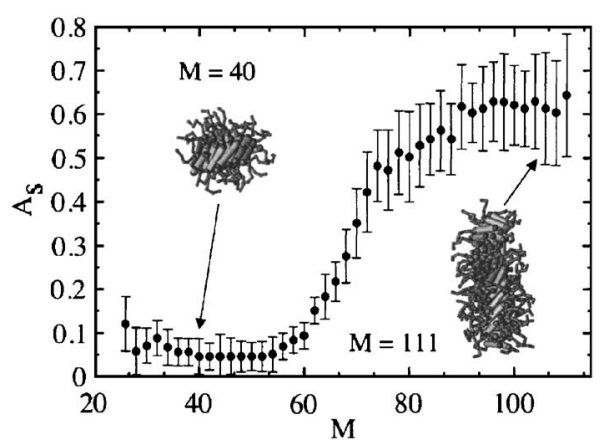

FIG. 5. Shapes of micelles obtained from a system of tethered rods at $\phi$ $=0.18$, and $1 / T=0.85$. The asphericity parameter is plotted as a function of the size, $M$, of the micelle. Individual micelles of size $M=40$ and $M=111$ are added as insets to aid the visualization of the asphericity parameter.

dominant and the micelles grow in a single dimension. We also postulate that because the core of the micelle is constructed of rigid rods the long micelles are rigid and therefore themselves act as rods resulting in a nematic phase.

For small system sizes, we find that the long micelles are tilted with respect to the coordinate axis. The tilt arises due to the finite size of the simulation box and may result in an artificial alignment of the long micelles. To ensure that the long micelles form a nematic phase and that this is not an artifact resulting from finite size effects, we employ the box search algorithm. ${ }^{24}$ Using this algorithm, we are able to obtain long micelles that are not tilted with respect to the coordinate axis. By comparing the energy and spacing of the tilted and nontilted structures, we find these values to be statistically identical and therefore we believe that the finite size of the box does not artificially influence the phase behavior and that the long micelles are a robust phase.

\section{Hexagonal chiral cylinder phase $(H)$}

For concentrations ranging from $\phi=0.2$ to $\phi=0.31$, we observe the formation of a hexagonal cylinder phase. Figure 6(a) is an example snapshot from a simulation of $N_{R}=6400$, $\phi=0.21$, and $1 / T=1.0$. Snapshots such as those in Fig. 6(b) reveal that within the cylinders the rods pack with a regular twist about the cylinder axis, forming cylinders that are chiral and nematically aligned. This is in contrast with previously assumed perfect parallel packing of the rods. ${ }^{26} \mathrm{Radz}$ ilowski et al. suggested that if the rods pack in parallel or in interdigitated bilayers or monolayers, they should form a square or rectangular cross section, which is not what they observed in computer reconstructions of their data. ${ }^{27}$ They suggested that this could be due to the rod-coil junctions not being confined to a planar surface. The twists of the rods observed in our simulations do not restrict the rod tether junction to a planar surface and as such the cross section is not square. Further evidence for this phase is demonstrated by the twisted ribbons observed in the work of Sone et al. in which short rod-coil copolymers form long twisted ribbons that are not mesoscopically ordered. ${ }^{28}$

Conventionally, chiral nematic structures form either from chiral building blocks or under the influence of an external field (25). The key to understanding how chirality arises in this system from the packing of building blocks that (a)
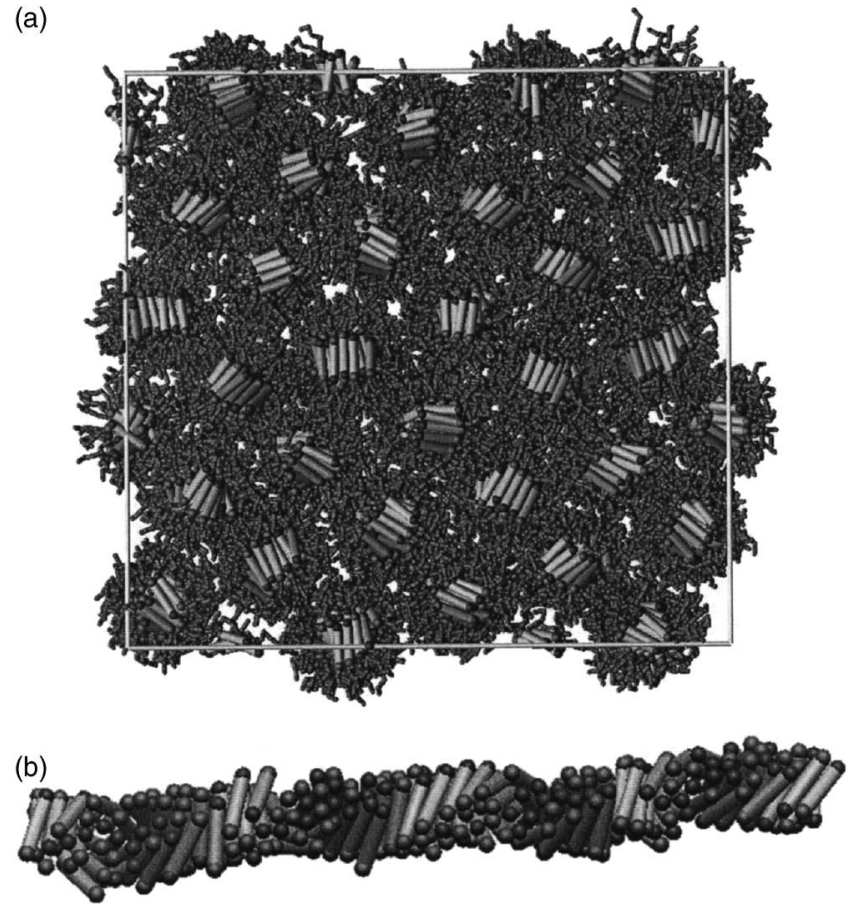

FIG. 6. Simulation snapshots obtained from a system of $N_{r}=6400$ tethered rods at $\phi=0.21$, and $1 / T=1.0$. (a) Snapshot of hexagonally ordered chiral cylinders. Rod ends with tethers attached are colored rod. Rod ends without tethers attached are colored blue. The periodic boundary conditions have been removed for visualization. (b) A single cylinder extracted from the simulation cell in "A." Again the rod ends that are attached to tethers are colored red. The rod ends that are not attached to tethers are colored blue. The tethers have been removed from the cylinder to view the local packing of the rods and the twist along the principle axis of the cylinder.

are, on average, achiral is again the competition between the interfacial energy and the elastic stretching energy of the flexible tethers. As suggested by Williams and Fredrickson, ${ }^{16}$ the interfacial energy of the sides of rod-coil cylinders is much lower than that of rod-coil junctions. Halperin argued ${ }^{14}$ and it has been shown experimentally ${ }^{9}$ that for twodimensional (2D) sheets the rods form a tilt angle with respect to the interfacial normal. This is believed to arise from the fact that the effective grafting density is lowered by increasing the distance between the anchor points of neighboring tethers. This argument should also apply to the cylinder phase but with the addition of a second angle. In liquid crystals, where chiral phases form from chiral molecules, the molecules do not pack parallel to neighboring molecules, but instead assume a slight tilt angle. ${ }^{29}$ Thus chirality in molecular liquid crystals is induced by a twist in the relative orientation of the chiral molecules, which propagates over macroscopic distances. In the tethered rod system, the tilt angle arises for entropic reasons associated with the excluded volume of the tether, inducing a twist that produces chiral selfassembled nanostructures. Additional entropy is gained by the rods aligning such that the tether anchor point is randomly on one side of the cylinder or the other, thereby decreasing the local grafting density of the tether. The random packing of the anchor points is illustrated by the colored spheres in Fig. 6(b).

Similar physics may underlie the recently discovered $B 2$ phase $^{30}$ comprised of achiral "banana-shaped" molecules as- 


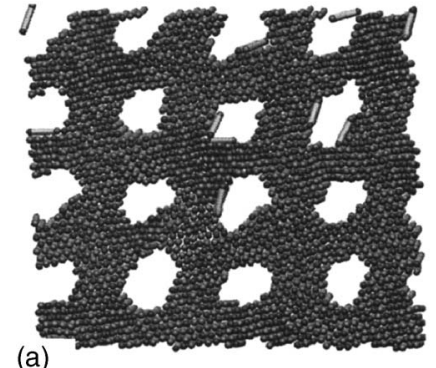

(a) (b)

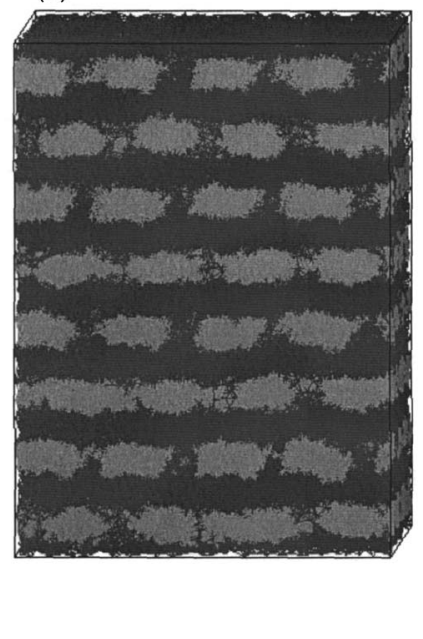

(c)

FIG. 7. Simulation snapshots of the tetragonally, perforated, lamellar morphology obtained from a system of $N_{r}=6400$ tethered rods at $\phi=0.34$; and $1 / T=0.75$. (a) A single sheet extracted from the simulation cell illustrating the square packing of the perforations within a layer. The tethers are removed to view perforations. The rod ends that have tethers attached are colored red. The rod ends that do not have tethers attached are colored blue. (b) A side view of the simulation cell. The rods are colored gold and the tethers are colored red. The rods and tethers are rendered as surfaces of constant density for visualization purposes. The discontinuities in the rod layers illustrate the $A B A B$ packing of holes between layers. (c) Snapshot of a single perforation extracted from the sheet in "A." The tethers are included to illustrate the packing of the tethers into the perforations.

sembled into chiral domains. ${ }^{30}$ In these experiments, oligomeric tails are linked to the end of the molecules, and the entropy of those tails may contribute to the tilt angle as they do in the simulations presented here. Thus, introducing functional groups or tethers to anisotropically shaped nanoparticles may provide a general method for creating chiral structures through self-assembly.

Since on average the tethered rods are not chiral, we expect that for a simulation cell of sufficient size we should find a racemic mixture - equal numbers of right and left handed cylinders. For small systems or systems where the cylinders are tilted with respect to the coordinate axis, there are not enough independent cylinders to determine if the mixture is indeed racemic. Again, we utilize the box search algorithm to obtain large systems where each simulation cell contains many individual cylinders. Data are taken from five independent simulations containing 30-36 independent cylinders per simulation cell. For an example simulation cell, see Fig. 6(a). Our simulations predict a slight bias in the systems toward right handed cylinders- $53 \%$ right handed as opposed to $47 \%$ left handed. We attribute this small difference to the finite size of the systems and argue that with larger systems sizes we would observe an equal number of right and left handed cylinders.

Additional finite size effects appear in the twist of the cylinders. The periodicity of the structures may not be exactly commensurate with the size of the simulation cell, and it is possible that the structures could deviate from the preferred morphologies to satisfy the periodic boundary condi-
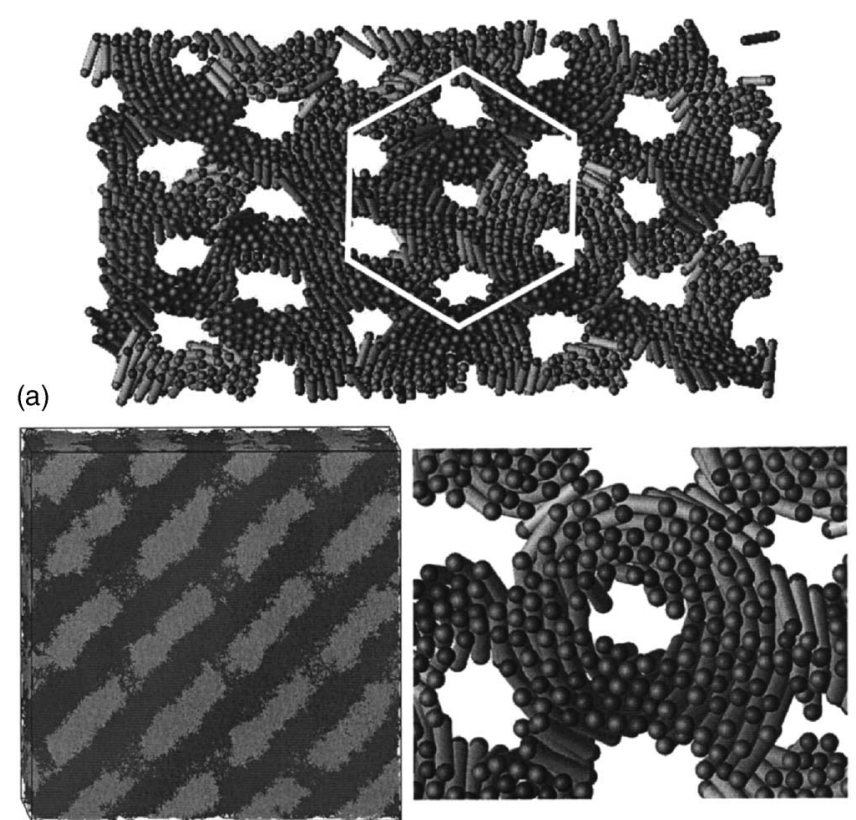

(b)

(c)

FIG. 8. Simulation snapshots of the hexagonally perforated lamellar phase obtained from a system of $N_{r}=4800$ tethered rods at $\phi=0.36$, and $1 / T$ $=0.75$. (a) A single sheet extracted from the simulation cell illustrating the hexagonal packing of the perforations within a layer. The tethers have been removed to enhance the viewing of the perforations. The rod ends that are attached to tethers are colored red. The rod ends that are not attached to tethers are colored blue. (b) Side view of simulation cell. The rods are colored gold and the tethers are colored red. The rods and tethers are rendered as surfaces of constant density for visualization purposes. The discontinuities observed in the rod layers illustrate the $A B A B$ packing of holes between layers. (c) Snapshot of a single perforation and its neighbors extracted from the sheet in "A." The tethers are removed to illustrate the packing of the tethers in the perforations.

tions. The box search algorithm is useful for minimizing finite size effects; however, because the twist along the cylindrical axis is periodic the hexagonally arranged chiral cylinders form a $3 \mathrm{D}$ periodic structure, which is difficult even for the box search algorithm to accommodate. Larger system sizes could be studied but they are computationally prohibitive because each building block contains multiple beads and because the time to self-assemble larger systems is longer because there are more particles to arrange. During the course of our simulations, we observe many systems with a discontinuity in the twist of the cylinder. The discontinuity may appear at any location along the cylinder. Immediately following the discontinuity, however, the twist resumes its regularity. Our simulations reveal, however, that this effect is small and that the chiral cylinders are robust. The tethered rods always self-assemble into the same cylindrical morphology with the same, intercylinder spacings, energy per particle, and twist rate-noting that for some systems there is a discontinuity in the twist.

We investigate to what extent the degree of the roughness of the rod impacts the twist rate. This is accomplished by spacing the spherical beads a distance $d$ apart as described in Sec. II. To this extent we perform simulations for rod beads spaced at $d=1.0,0.5$, and $0.25 \sigma$, consisting of $N_{r b}=5$, 9 , and 17, at $\phi=0.21$ for three independent systems of size $N_{R}=6400$. We note that the effective rod-rod interaction 
(a)

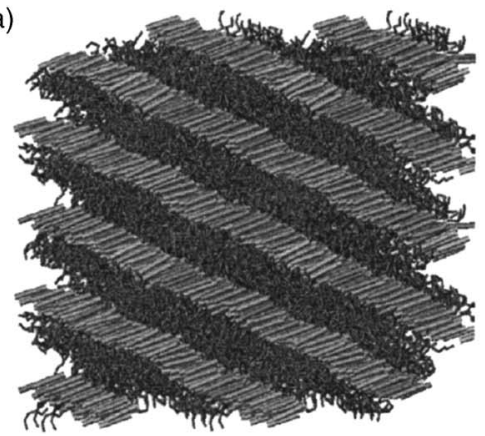

(c)

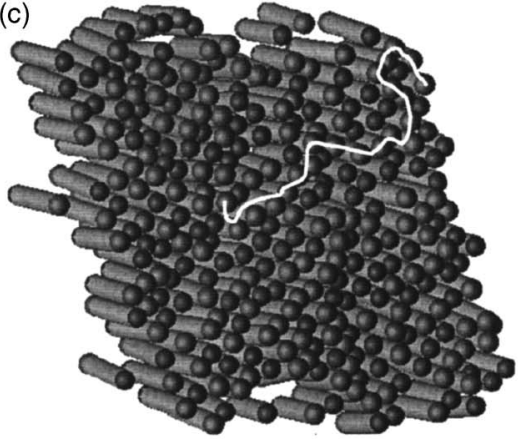

(b)

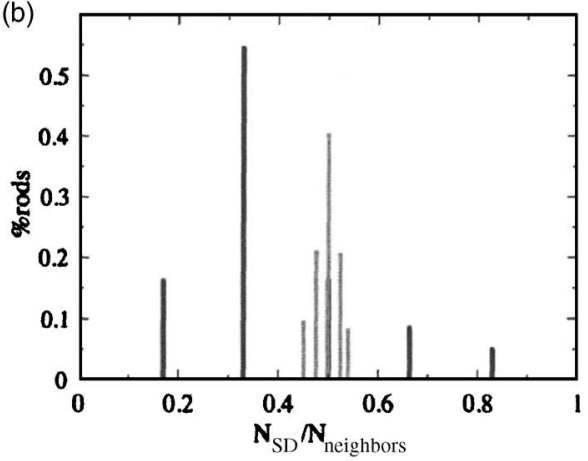

(d)

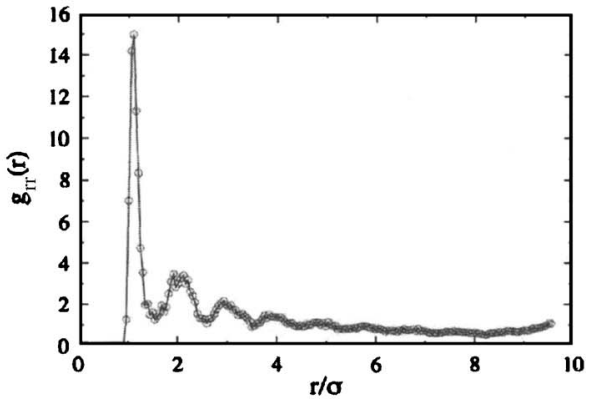

FIG. 9. Simulation snapshots of the smectic C lamellar phase obtained from a system of $N_{r}=4800$ tethered rods at $\phi=0.42$, and $1 / T=0.5$. (a) Side view of simulation cell. The rods are colored gold and the tethers red. (b) Distribution of percentage of neighboring rods pointing in the same direction. The red histogram considers only first neighbors and the green histogram considers up to third neighbors. (c) Single sheet extracted from the simulation cell in "A." The tethers have been removed for viewing ease and the rod ends attached to tethers are colored red and those without tethers colored blue. This coloring scheme is used to highlight the stringlike patterns predicted by the distribution in "B." (d) Plot of the rod-rod pair correlation function. strength was scaled to account for the larger number of interaction sites over the same length rod. No apparent change in the rate of twist was observed, providing strong evidence that the twist does not result from inter-rod interactions nor is the twist a function of the smoothness of the rod. To further investigate the cause of the twist, we performed simulations in which the degree of polymerization of the tether was varied and these simulations are discussed in Sec. IV.

\section{Tetragonally and hexagonally perforated lamellar phases}

Our simulations predict that from $\phi=0.31$ to $\phi=0.36$ the tethered rods from 3D tetragonally and hexagonally perforated lamellar phases, respectively. Both these structures have been reported experimentally. ${ }^{10,11}$ However, neither theory nor previous simulations have predicted the formation of these phases for PTR, presumably due to the assumptions of perfect ordering of the rigid rods, the initial guess of the candidate structure, or the small number of dimensions considered. These two complex 3D structures result from the competition between the rods attempting to maximize their contact with other rods to minimize energy and the tethers attempting to maximize their free volume to maximize entropy. As the concentration of the system is increased from that of the cylinder phase, the repulsive interactions between aggregates induce a morphology change toward aggregates with a higher surface area to volume ratio. Figure $7(\mathrm{a})$ is an example simulation snapshot for a system of 6400 tethered rods, at $\phi=0.34$, and $1 / T=0.75$. Snapshots of the tetragonally perforated lamellar phase reveal that the rods pack into monolayer sheets with holes ordered into a square lattice [see Fig. 7(b)]. The snapshots also reveal that the rods pack within the sheet such that their long axis is parallel to the interfacial normal of the sheet. In addition to ordering within a sheet, the perforations order such that between sheets the perforations from an $A B A B$ stacking resulting in 3D ordering of the perforations [see Fig. 7(c)]. Packing the rods into sheets is highly favorable for the rods but depending on the packing is not favorable for the tethers. To minimize the elastic strain, the rods pack in monolayers with the tether anchor points distributed randomly on both sides of the sheet. Additionally, by forming perforations the effective grafting density of the tethers is decreased because the tethers can occupy the perforations. The diameter of the perforations is on the order of twice the average end-to-end distance of the tethers. A snapshot of a perforation, shown in Fig. 7(c), illustrates that the perforations are filled with tethers. As the concentration is further increased, the additional rods can either occupy the perforations keeping the spacing between the sheets the same or the spacing between the sheets can decrease and the sheets can remain perforated. Interestingly, we observe the formation of a hexagonally perforated lamellar phase. Figure 8(a) is a snapshot of a simulation cell for 4800 rods, at $\phi=0.36$ and $1 / T=0.75$. As in the tetragonally perforated lamellar phase, the perforations between the sheets pack such that they form an $A B A B$ pattern resulting in $3 \mathrm{D}$ ordering of the perforations [see Fig. 8(b)]. The diameter of the holes is again on the order of twice the end-to-end distance of the tether. By ordering in a hexagonal pattern, the density of the perforations can be increased. In contrast to the tetragonally perforated sheets, the rods within the hexagonally perforated sheets are not parallel to the interfacial normal and there appears to be a handedness in the packing of the rods around a perforation [see Fig. 8(c)]. By tilting, the rods can further reduce the elastic strain of the tethers at a penalty of decreasing the rod-rod contacts.

\section{E. Smectic C lamellar phase}

As the concentration is increased further, $\phi>0.36$, there is a transition from the perforated lamellar sheets to a smec- 
TABLE I. Measured tilt angle of the smectic $\mathrm{C}$ phase and the temperatures corresponding to the angles.

\begin{tabular}{cc}
\hline \hline$T\left(\varepsilon / k_{B} T\right)$ & Tilt angle (smectic C) \\
\hline 2 & $37^{\circ}$ \\
2.2 & $39^{\circ}$ \\
2.5 & $40^{\circ}$ \\
2.8 & $42^{\circ}$ \\
3.3 & $43^{\circ}$ \\
\hline \hline
\end{tabular}

tic C lamellar phase. Figure 9(a) is an example of a simulation snapshot of 4800 tethered rods, at $1 / T=0.5$ and $\phi=0.42$. This snapshot reveals that the rods form a tilt angle with respect to the interfacial normal. Unlike smectic C phases observed for systems of hard rods, a layer of polymer tethers separates the smectic layers. It has been argued that the tilt angle arises due to a competition between the rods attempting to maximize their contact with other rods and the flexible tethers attempting to maximize their entropy or free volume. ${ }^{14,31}$ Semenov and Vasilenko's scaling analysis predicts an increase in the tilt angle with an increase in temperature. $^{32}$ Our simulations quantitatively demonstrate the $T$ dependence of the tilt angle in the smectic $\mathrm{C}$ phase (see Table I). For example, we find that for an increase in temperature from $T=2.0$ to $T=3.3$, there is an increase in the tilt angle from $\theta=37^{\circ}$ to $\theta=43^{\circ}$, which is consistent with theoretical predictions. As illustrated in Fig. 9(a), the rods form a monolayer sheet and not a bilayer as observed for other surfactant systems. ${ }^{3,33}$ In the case of the tethered rods, there is a large entropic penalty for forming a bilayer because the rod packing would be such that the neighbors of each rod would have their tethers on the same side of the bilayer. This entropic penalty cannot be offset by the small energetic benefit of packing the rods end to end. The packing of the rods within the monolayer sheets can be described by the distribution of rods and the number of neighboring rods with tethers on the same side of the sheet. Figure 9(b) shows the percentage of rods plotted against the percentage of neighbors with their anchor points on the same side of the sheet. When only the first neighbors are considered, our simulations reveal that more than $50 \%$ of the rods in the system have only $1 / 3$ of their neighbors with tethers on the same side of the sheet. Since on average each rod has six first neighbors the distribution of tether anchor points results in a coordination number of 2 . This corresponds to chainlike patterns of the anchor points within the sheets. Figure 9(c) is a single sheet extracted from the simulation snapshot in Fig. 9(a) and the tethers are removed for viewing ease. To illustrate the chainlike patterns we highlight the rod ends with tether anchor points in red and those without in blue. This snapshot clearly illustrates the chainlike patterns within the sheet. In addition in Fig. 9(b), we plot the distribution of anchor points considering the first and third neighbors. This plot illustrates that as the radius of inclusion of neighboring particles increases, the probability that given rod neighbor have a tether anchor point on the same side of the sheet approaches $50 \%$. In a smectic $\mathrm{C}$ phase there exists no longrange order within a sheet. The lack of long-range order

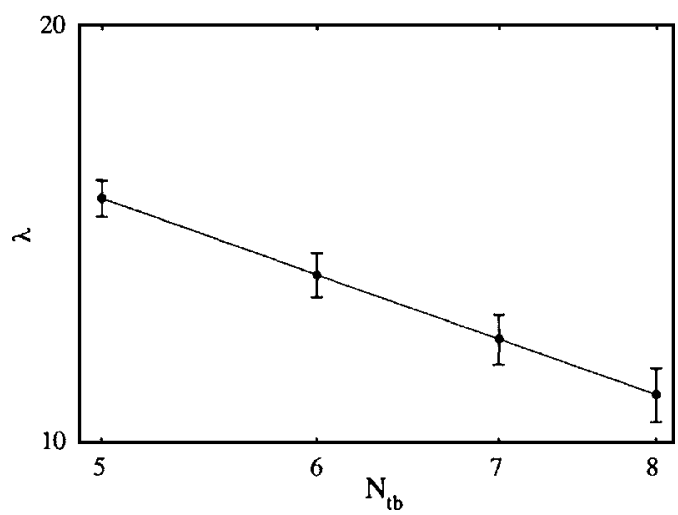

FIG. 10. Plot of the degree of polymerization of the tether $\left(N_{t b}\right)$ vs the wavelength $(\lambda)$ of the twist observed for the chiral cylinder phase.

within a sheet in the smectic $\mathrm{C}$ lamellar phase can be demonstrated by the rod-rod pair correlation function, Fig. 9(c). The correlation function demonstrates several peaks characteristic of hexagonal packing but decays rapidly and beyond several rod diameters there is no correlation.

\section{EFFECTS OF RELATIVE COIL FRACTION}

To study the effect of the degree of polymerization of the tethers simulations of systems of size $N_{R}=1000$ and 6400 at $\phi=0.21$ are run. The degree of polymerization of the tether is varied from $N_{t b}=1-10$. For systems where the degree of tether polymerization is between 5 and 8 , the hexagonally arranged chiral cylinder structure remained intact. However, for degrees of polymerization outside this range, we observe a change in the equilibrium morphology. Two effects occur as the length of the tether is changed. The first effect is that the degree of compressibility of the soft matter matrix changes. For example, as the size of the tether is increased the soft shell formed by the tethers around the aggregate becomes more compressible; that is, there is effectively less repulsion between aggregates and less desire to form aggregates with high surface area to volume ratios. The second change is the degree of curvature induced by the length of the tether. For example, as the length of the tether is decreased the driving force to form morphologies with curved interfaces decreases. These two effects are discussed in subsequent paragraphs.

We first discuss the case where the degree of polymerization does not result in a change in the structural morphology. As the size of the tether is increased from $N_{t b}=5$ to $N_{t b}=8$, we observe an increase in the rate of twist. To quantify this effect we plot the wavelength of the twist, $\lambda$, versus the size of the tether $N_{t b}$. Figure 10 is a $\log -\log$ plot of $\lambda$ versus $N_{t b}$ and illustrates a linear decrease in the wavelength with an increase in the degree of polymerization. Therefore as the tether increases in length, it becomes increasingly more difficult for the rods to pack parallel to each other within a cylinder and the interfacial curvature increases. As the degree of polymerization is further increased such that $N_{t b}$ is larger than 8 the cylinders break apart and micelles are formed. It could be argued that the formation of the micelles does not result from the desire of the tethers to increase the interfacial curvature but that as previously mentioned it may 

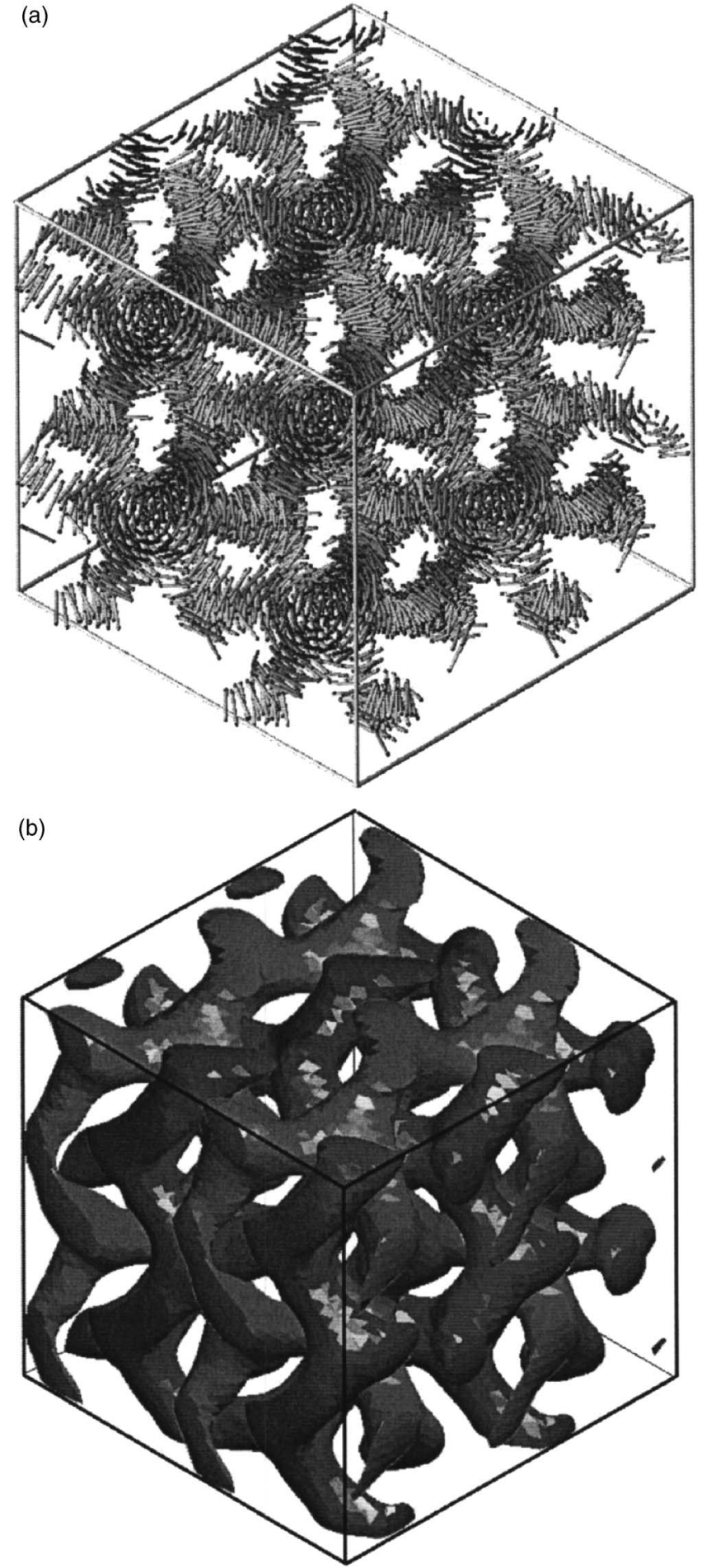

FIG. 11. Simulation snapshots obtained from a system of $N_{r}=800$ tethered rods at $\phi=0.21$, and $1 / T=1.0$. (a) Simulation snapshot illustrating the gyroid phase. For visualization of the underlying structure the system has been replicated, the diameter of the rods has been decreased, and the tethers have been removed. In this system, the rod is of length 5 and the tether of length 2. Rod ends attached to tethers are colored red and those without tethers are colored blue. (b) Simulation snapshot as in "A," where the rods have been rendered as surfaces of constant density. The red and blue surfaces illustrate the two interconnected networks and the tethers have been removed for viewing ease.

result from the soft-shell around the aggregate becoming more compressible. However, the micelles that are formed are elongated and ordered into columns that are hexagonally

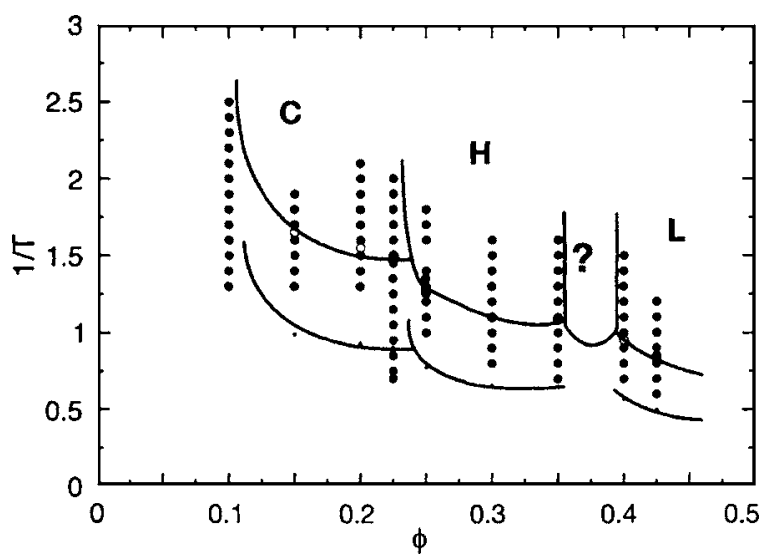

FIG. 12. Temperature vs concentration phase diagram for small aspect ratio tethered rods. The curves do not represent actual calculated phase boundaries but are used as guides to identify the areas in which a particular ordered phase is observed. $\mathrm{C}, \mathrm{H}$, and $\mathrm{L}$ represent the bcc ordered micelles, the hexagonal cylinder morphology, and the smectic $\mathrm{C}$ lamellar morophology, respectively. The question mark indicates a region where we are unable to determine the equilibrium structure. We suspect that the gyroid phase may be stable here. The blue curves represent phase boundaries that have been rescaled to account for the size of the tethered rods.

arranged. The ordering of the micelles suggests that there are strong intermiceller interactions and it is therefore likely that the driving force to form micelles stems from the desire of the tethers to increase their interfacial curvature. Decreasing the tether length provides further evidence that it is the tethers that are driving the curvature effects observed in the chiral cylinders. As the tether size is decreased from $N_{t b}=5$ to $N_{t b}=2$ and then to $N_{t b}=1$ the system undergoes two phase changes. For $N_{t b}=2$ at $\phi=0.21$, the system forms the double gyroid phase and for $N_{t b}=1$ the system forms a monolayer sheet. Figures 11(a) and 11(b) are example snapshots of the gyroid phase. Therefore, as the tether size decreases structures with less and less curvature form as indicated by the transition from hexagonally packed cylinders, to the gyroid structure and then to a lamellar sheet with no curvature. This pattern suggests that as the tether size decreases the rod-rod interactions dominate and their desire to form a flat interface determines the final structure. For $N_{t b}=3$ and $N_{t b}=4$ there appears to be a competition between the cylinder and the gyroid phase and only interconnected tubes are observed in the simulations.

\section{SELF-ASSEMBLY OF SMALL ASPECT RATIO TETHERED RODS}

For hard rod systems the rod aspect ratio significantly influences the types of structures the rods form. ${ }^{25}$ However, it is not clear to what extent the aspect ratio affects the selfassembled structures in rods that are permanently connected to a flexible tether and which have attractive interactions between rods. To this extent, we simulated a system of tethered rods where the aspect ratio of the rod is 3:1. To compare with the above system, we study a system that is symmetric with regard to the size. That is, the tether and the rod are of the same size. In this section we discuss the $T$ versus $\phi$ phase behavior of small aspect ratio rods plotted in Fig. 12. The lower curves represent phase boundaries that have been 
(a)

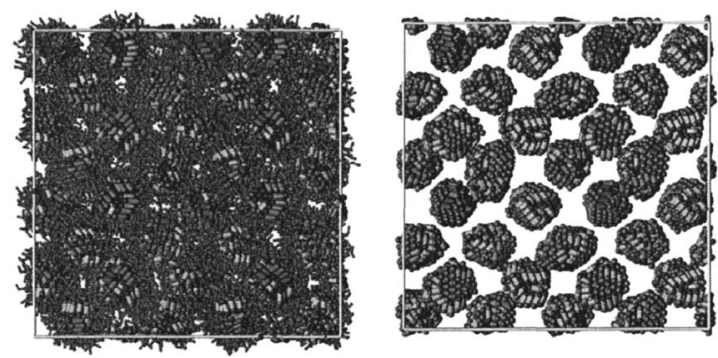

(b)

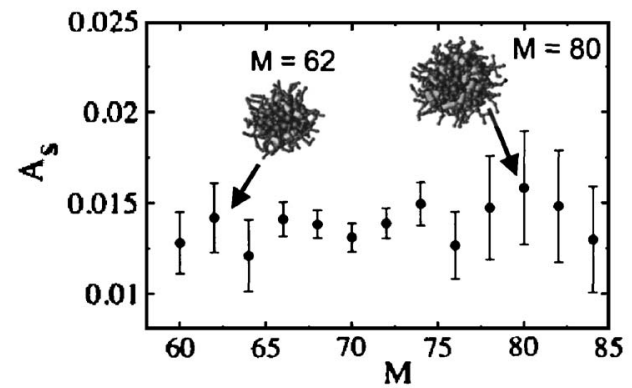

(c)
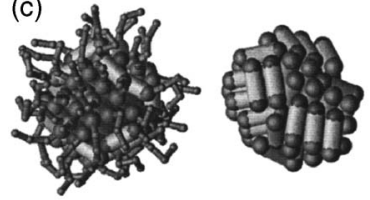

(a)
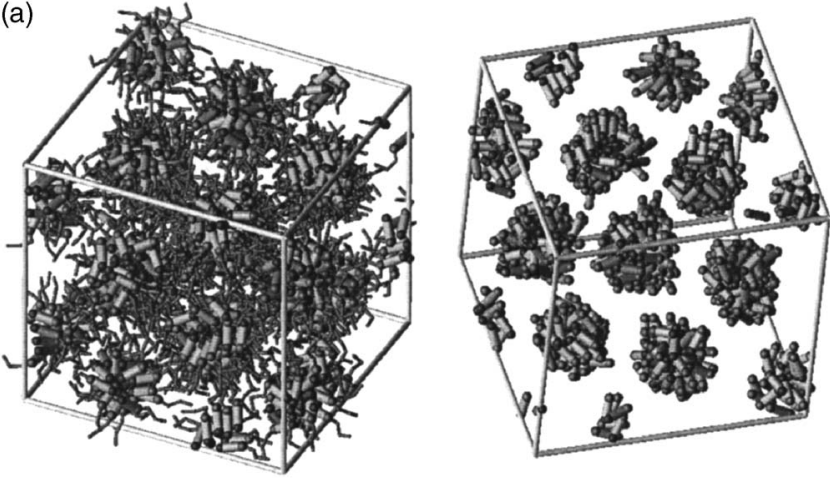

(b)

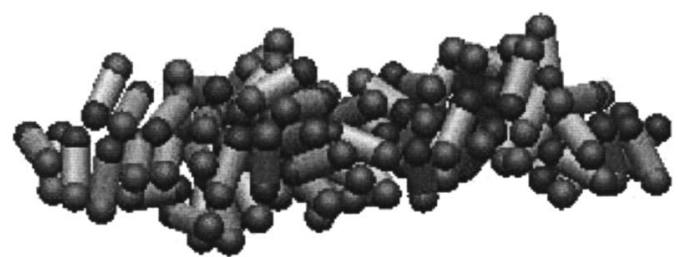

FIG. 14. Simulation snapshots of the hexagonal cylinder phase obtained from $N_{r}=1600$ tethered rods at $\phi=0.3$. (a) Snapshot of the simulation cell with tethers (left) and without tethers (right). Rod ends attached to tethers are colored red and those that are not attached to tethers are colored blue. (b) Single cylinder extracted from the simulation cell in "A." The tethers have been removed to visualize the packing of the rods.

FIG. 13. Simulation snapshot of the bcc micelle phase formed from $N_{r}$ $=4800$ tethered rods of small aspect ratio at $\phi=0.21$, and $1 / T=2.0$. (a) Snapshot of simulation cell of bcc ordered micelles with tethers (left) and without tethers (right). (b) Shape of micelles. The asphericity parameter is plotted as a function of the size of the micelle, $M$. Example micelles are included to illustrate the value of the asphericity parameter. (c) Individual micelle of size $M=62$ (far left), extracted from the simulation cell in "A." The tethers extend radially from the center of the micelle. (Left) same micelle with tethers removed. The core of the micelle is spherical in shape (Right) single micelle of size $M=80$, extracted from the simulation cell in "A." Again the tethers extend radially from the micelle. (Far right) same micelle with tethers removed. The core of the micelle is spherical in shape.

scaled to account for the disparity between the moderate aspect ratio rods and the low aspect ratio rods. This scaling is analogous to using the $\chi N$ parameter commonly used to describe the inverse temperature of block copolymers. That is, the inverse temperature is just rescaled by the ratio of the number of beads in the moderate aspect ratio rods to the number of beads in the low aspect ratio rods.

\section{A. Cubic micelle phase $(C)$}

For $0.1 \leqslant \phi<0.25$, the rods self-assemble into micelles which arrange into a bcc morphology. Figure 13(a) is a snapshot of a simulation cell containing 4800 tethered rods, at $1 / T=2.0$, and $\phi=0.21$. Simulation snapshots reveal that in contrast to the moderate aspect ratio rods no nematically ordered long micelle phase forms and the bcc ordered micelles persist to higher concentrations. To quantify the shape of the micelles, the asphericity parameter is plotted as a function of the size of the micelle [Fig. 13(b)]. There is a lack of significant change in $A_{s}$ over the size range of observed micelles and because $A_{s}<0.1$, we conclude that the shape of the micelle is roughly spherical and only weakly depends on the number of rods within the micelle. Simulation snapshots reveal that within the micelles, the rods do not have a specific direction along which they align, as in the case for the high aspect ratio rods. Figure 13(c) shows individual micelles extracted from the simulation cell illustrated in Fig. 13(a). Here the tethers are oriented to extend radially from the center of the micelle and the overall micelle is spherical in shape as indicated by $A_{s}$. As illustrated in Fig. 13(c), the micelles appear to be spherical without the tethers due to the lack of directional order of the rods within the micelle, unlike in the case of moderate aspect ratio rods. Also, unlike the moderate aspect ratio rods, the micelles are spherical in shape over a broad size range and the intermicellar interactions are effectively isotropic. This suggests that it is the isotropic shape of the core that allows the micelles consisting of small aspect ratio rods to maintain their spherical shape and thus form a bcc morphology over a broader concentration range than the moderate aspect ratio rods.

\section{B. Hexagonal cylinder phase}

As the concentration is increased such that $\phi>0.25$, the repulsive interactions between micelles become significant and there is a need to rearrange into structures with a greater surface area to volume ratio such as the hexagonal cylinder phase. Our simulations reveal that for $0.25 \leqslant \phi<0.36$, the hexagonal cylinder phase is stable. Figure 14(a) is a snapshot of a simulation cell containing 1600 tethered rods. In contrast to the cylinder phase observed for moderate aspect ratio tethered rods, to a large extent the rods pack in the cylinders with their tether-free ends pointing in toward the center of the cylinder and the rods are not highly oriented [see Figs. 14(a) and 14(b)]. The most striking observation, however, is the lack of the chirality observed in this system [see Fig. 14(b) ] as compared to the moderate aspect ratio rod system. We postulate that because the rods have a small aspect ratio 
there is only a weak desire to align and therefore the entropic forces contributed by the tether dominate and the rods pack randomly. Another difference that arises due to a lack of alignment between neighboring rods is that neighboring rods do not alternate in direction but that in general they are oriented in a radial direction from the center of the cylinder. For the moderate aspect ratio rods, it could be argued that the chirality results from the mesoscopic packing of the cylinders and that the handedness results in an effective decrease in concentration. However, our simulations reveal that the hexagonal cylinder phase persists at higher concentrations for systems containing the small aspect ratio rods. Since the tethers are shorter in the case of the small aspect ratio rods, they are to some extent less compressible. Therefore, we postulate that if the driving force to form chiral cylinders arose due to packing on the mesoscopic level the small aspect ratio rods would align and form chiral cylinders. That is, we propose that the chirality arises as result of the complex balance between the moderate aspect ratio rods desire to align and the subsequent entropic penalty suffered for that alignment.

\section{Smectic C lamellar phase}

For concentrations between those where cylinders and lamellar phases are formed, we observe only the formation of bicontinuous structures consisting of interconnected tubes. For these concentrations the tethered rods may be trying to form the gyroid phase but we have thus far been unsuccessful in observing this structure in the short rod case. The role of the rod length in stabilizing the gyroid phase will be explored in a different paper. For $\phi>0.38$, the repulsive forces between aggregates dominate and there is a morphological change to a smectic $\mathrm{C}$ lamellar phase. By forming the sheetlike phase the spacing between aggregates is maximized. Figure 15(a) is a snapshot from a system of 1600 tethered rods, at $\phi=0.4$, and $1 / T=1.25$. The snapshot reveals that the rods form a slight tilt angle with respect to the interfacial normal forming a smectic $\mathrm{C}$ phase where the smectic layers are separated by a layer of tethers. Within the layers, the simulations show that there is no long-range order similar to the case of the moderate aspect ratio rods. However, although the structures look similar in that the rods are aligned and organized into smectic $\mathrm{C}$ layers there is a significant difference in the ODT temperature and the tilt angle of the rods between the moderate and small aspect ratio rods. For systems where the inverse temperature is below $1 / T=1.0$, the system does not form a smectic phase but the rods pack randomly within the sheets as illustrated in Fig. 15(b). The ODT temperature for the moderate aspect ratio rods is approximately 4.0, while it is only 2.0 for the small aspect ratio rods. From this perspective, the small aspect ratio rods act as flexible chains. That is, their desire to align is weak in contrast to the moderate aspect ratio rods and the entropic forces contributed by the tether dominate the final ordered structure.

\section{DISCUSSION}

The interplay between liquid crystal ordering and microphase separation can create new structures in self-

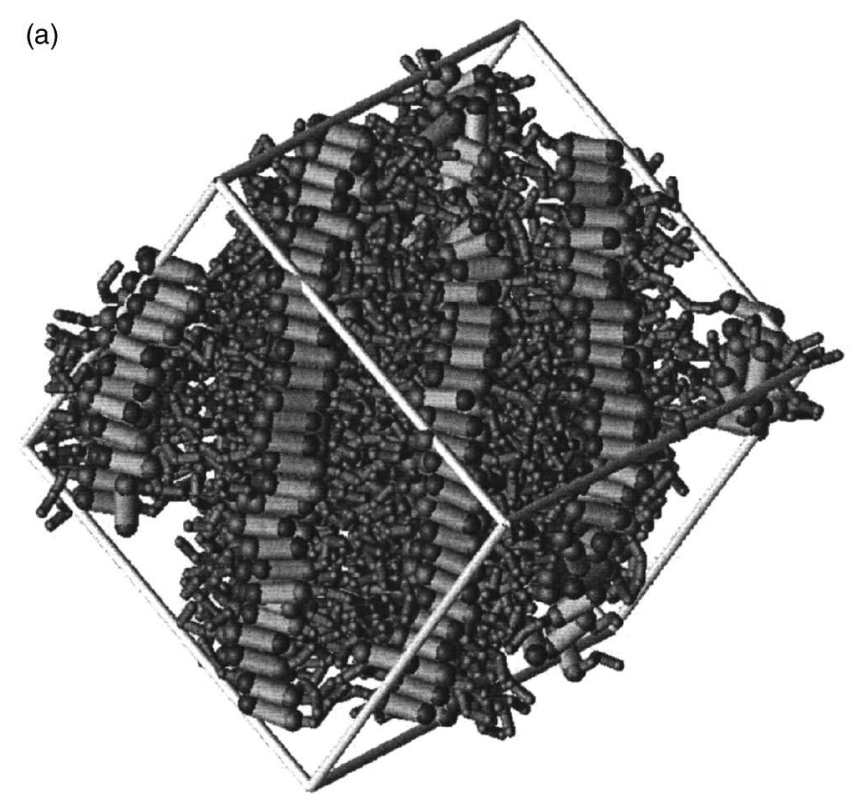

(b)

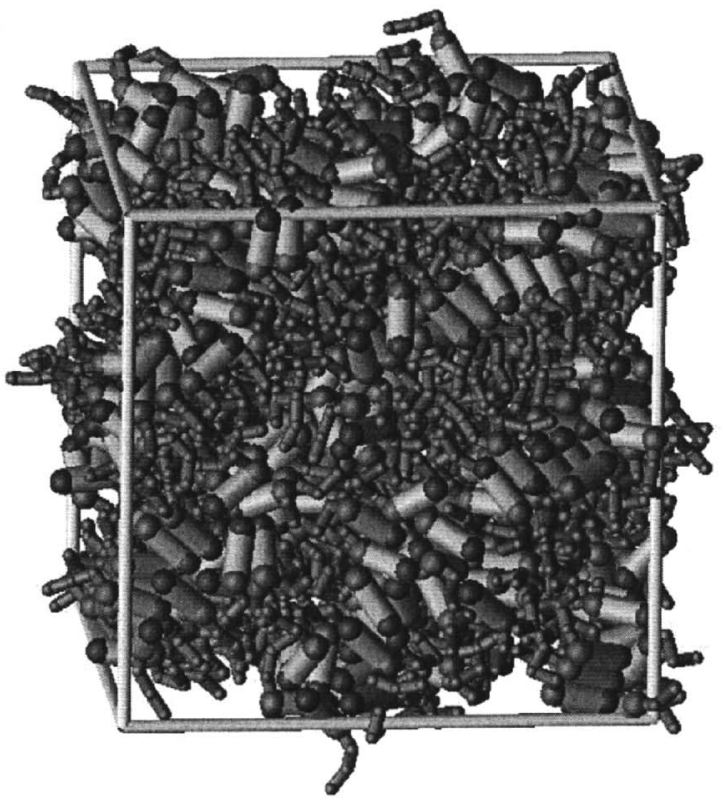

FIG. 15. Simulation snapshots of the smectic C lamellar phase obtained from $N_{r}=1600$ small aspect ratio rods at $\phi=0.4$. (a) Simulation snapshot for $1 / T=1.25$. Diagonal view illustrates the smectic layers. (b) Simulation snapshot for $1 / T=1.0$. This temperature is very close to the ODT temperature and the snapshot reveals that the rods are only moderately aligned.

assembled nanoparticle-based shape amphiphiles. For tethered nanorods, the competition between the interfacial energy and the elastic energy of the polymer tethers is key to understanding the various assemblies. Depending on the mesophases induced by microphase separation, this competition is manifested differently, resulting in a variety of structures. For moderate aspect ratio rods, the desire of the rods to align combined with the desire of the tether to maximize its free volume result in rich structures such as the nematically ordered long micelle phase, the chiral cylinder phase, and the perforated phases. In contrast, for small aspect ratio rods there is only a weak desire for the rods to align and the structures are dominated by the desire of the tether to maximize its free volume while maintaining the optimal spacing 
between rods. Therefore, the observed structures, in general, possess only mesoscale ordering and very little local ordering. Further, for the moderate aspect ratio rods the degree of polymerization of the tether provides a systematic way in which both the local and mesoscale orderings of a structure can be modified in a somewhat predictable manner. That is, if the tether length is decreased the rods increasingly form structures with less interfacial curvature. The success of this model in predicting experimentally observed structures that have not been predicted via theory suggests that this simple model may be useful in predicting the phase behavior of polymer-tethered building blocks with more complicated topologies and geometries, ${ }^{2}$ and, in particular, may be useful in guiding the design of building blocks that can form chiral structures. Further, the success of this model in predicting the phase behavior of tethered rod systems indicates that, to some extent, this behavior is universal. The most important parameters controlling the equilibrium structure-aspect ratio, degree of polymerization, interaction strength, and system concentration-can be controlled experimentally.

\section{ACKNOWLEDGMENTS}

The authors thank R. G. Larson, C. R. Iacovella, and E. R. Jankowski for helpful discussions. In addition, they thank C. R. Iacovella for assistance with rendering the images. Financial support for this work was provided by the U.S. Department of Energy, Grant No. DE-FG02-02ER46000.

${ }^{1}$ G. M. Whitesides and B. Grzybowski, Science 295, 2418 (2002).

${ }^{2}$ Z. L. Zhang, M. A. Horsch, M. H. Lamm, and S. C. Glotzer, Nano Lett. 3, 1341 (2003).

${ }^{3}$ C. R. Iacovella, M. A. Horsch, Z. Zhang, and S. C. Glotzer, Langmuir 21, 9488 (2005).

${ }^{4}$ M. A. Horsch, Z. L. Zhang, and S. C. Glotzer, Phys. Rev. Lett. 95, 056105 (2005).

${ }^{5}$ E. R. Chan, X. Zhang, C. Y. Lee, M. Neurock, and S. C. Glotzer, Macromolecules 38, 6168 (2005); S. C. Glotzer, M. A. Horsch, C. R. Iacovella, Z. L. Zhang, E. R. Chan, and X. Zhang, Curr. Opin. Colloid Interface Sci. 10, 287 (2005)
${ }^{6}$ Z. L. Zhang and S. C. Glotzer, Nano Lett. 4, 1407 (2004).

${ }^{7}$ C. Tschierske, J. Mater. Chem. 11, 2647 (2001).

${ }^{8}$ J. W. Park and E. L. Thomas, Macromolecules 37, 3532 (2004).

${ }^{9}$ J. T. Chen, E. L. Thomas, C. K. Ober, and S. S. Hwang, Macromolecules 28, 1688 (1995).

${ }^{10}$ M. Lee, B. K. Cho, K. J. Ihn, W. K. Lee, N. K. Oh, and W. C. Zin, J. Am. Chem. Soc. 123, 4647 (2001).

${ }^{11}$ N. K. Oh, W. C. Zin, J. H. Im, J. H. Ryu, and M. Lee, Chem. Commun. (Cambridge) 2004, 1092.

${ }^{12}$ M. Lee, B. K. Cho, Y. S. Kang, and W. C. Zin, Macromolecules 32, 7688 (1999).

${ }^{13}$ S. A. Jenekhe and X. L. Chen, Science 279, 1903 (1998).

${ }^{14}$ A. Halperin, Europhys. Lett. 10, 549 (1989).

${ }^{15}$ A. N. Semenov and S. V. Vasilenko, Zh. Eksp. Teor. Fiz. 90, 124 (1986).

${ }^{16}$ D. R. M. Williams and G. H. Fredrickson, Macromolecules 25, 3561 (1992).

${ }^{17}$ R. Holyst and M. Schick, J. Chem. Phys. 96, 730 (1992).

${ }^{18}$ M. W. Matsen, J. Chem. Phys. 113, 5539 (2000).

${ }^{19}$ V. Pryamitsyn and V. Ganesan, J. Chem. Phys. 120, 5824 (2004).

${ }^{20}$ J.-Z. Chen, C.-X. Zhang, Z.-Y. Sun, Y.-S. Zheng, and A. Li-Jia, J. Chem. Phys. 124, 104907 (2006).

${ }^{21}$ M. Lee, B. K. Cho, and W. C. Zin, Chem. Rev. (Washington, D.C.) 101, 3869 (2001)

${ }^{22}$ G. S. Grest and K. Kremer, Phys. Rev. A 33, 3628 (1986).

${ }^{23}$ M. P. Allen and D. Tildesley, Computer Simulations of Liquids, 1st ed. (Oxford University Press, New York, 1987).

${ }^{24}$ A. J. Schultz, C. K. Hall, and J. Genzer, J. Chem. Phys. 120, 2049 (2004).

${ }^{25}$ P. Bolhuis and D. Frenkel, J. Chem. Phys. 106, 666 (1996).

${ }^{26}$ H. A. Klok and S. Lecommandoux, Adv. Mater. (Weinheim, Ger.) 13, 1217 (2001).

${ }^{27}$ L. H. Radzilowski, B. O. Carragher, and S. I. Stupp, Macromolecules 30, 2110 (1997).

${ }^{28}$ E. D. Sone, E. R. Zubarev, and S. I. Stupp, Angew. Chem., Int. Ed. 41, 1706 (2002).

${ }^{29}$ R. D. Kamien and J. V. Selinger, J. Phys.: Condens. Matter 13, R1 (2001).

${ }^{30}$ A. Jakli, D. Kruerke, and G. G. Nair, Phys. Rev. E 67, 051702 (2003); D. R. Link, G. Natale, R. Shao, J. E. Maclennan, N. A. Clark, E. Korblova, and D. M. Walba, Science 278, 1924 (1997); T. Niori, T. Sekine, J. Watanabe, T. Furukawa, and H. Takezoe, J. Mater. Chem. 6, 1231 (1996); J. Xu, R. L. B. Selinger, J. V. Selinger, and R. Shashidhar, J. Chem. Phys. 115, 4333 (2001).

${ }^{31}$ A. Halperin, Macromolecules 23, 2724 (1990).

${ }^{32}$ A. N. Semenov, Mol. Cryst. Liq. Cryst. 209, 191 (1991).

${ }^{33}$ R. G. Larson, J. Chem. Phys. 89, 1642 (1988). 